\title{
Fluorescence Decay Dynamics of Ethidium Bromide in Polymers
}

\author{
Ah-Young Jee and Minyung Lee*

\begin{abstract}
Division of Chemistry and Nanoscience, Ewha Womans University, Seoul 120-750, Korea. *E-mail: mylee@ewha.ac.kr Received April 2, 2010, Accepted May 10, 2010
\end{abstract}

Key Words: Ethidium bromide, Fluorescence lifetime, Dynamics, Elastic modulus

The medium influence on the fluorescence decay dynamics of ethidium bromide (EB) has been investigated in various environments. For example, Ohmstead and Kearns related the fluorescence lifetime of EB to the excited-state proton transfer process. ${ }^{1}$ In addition, they reported that the solvent viscosity plays a minor role in the excited state decay process of EB. Chirico et al. measured the fluorescence decay of EB as $1.7 \mathrm{~ns}$ in water and $6.5 \mathrm{~ns}$ in ethanol and concluded that hydrogen bonding ability is a key factor for the nonradiative relaxation. ${ }^{2}$ Pal et al. measured the fluorescence decay time of EB in acetone, acetonitrile, and their mixtures. They observed that the fluorescence decay processes were independent on the solvent polarity. ${ }^{3}$ These results show that the EB lifetime does not depend much on polarity or viscosity, but is mainly influenced by hydrogen bonding. Overall, EB is one of most widely used dyes for probing DNA. When EB is intercalated into the helical structure of DNA, a large increase in the fluorescence lifetime has been observed in comparison with water environment, and the fluorescence enhancement was attributed to the blocking of the excited-state proton transfer. ${ }^{4,5}$

The main objective of this work is to investigate whether the fluorescence decay dynamics of EB is influenced by the environmental rigidity of polymer. As shown in Figure 1, EB contains a phenyl ring the motion of which may play a role in the rate processes of the electronically excited EB. We have measured the fluorescence lifetime of EB in various polymers including low density polyethylene (LDPE), high density polyethylene (HDPE), polycarbonate (PC), polystyrene (PS), and polyacrylic acid (PAA). We observed that the fluorescence lifetime of EB is strongly correlated to the Young's modulus of polymer and, based on the observed fluorescence lifetimes, we attempted to delineate the nonradiative decay processes of EB in terms of polymer rigidity.

EB was obtained from Invitrogen. LDPE (MW 35,000), HDPE (MW 125,000), PC (MW 64,000), PS (MW 192,000), and PAA (MW 240,000) were purchased from Sigma-Aldrich. Different solvents were used to prepare the polymer solutions: tetrahydrofuran (THF) for LDPE and PC; decalin for HDPE; cyclohexane for PS; water for PAA. All films for the lifetime

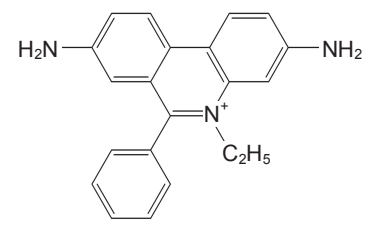

Figure 1. The molecular structure of ethidium bromide. measurements were prepared by spin coating and dried in an oven at $70{ }^{\circ} \mathrm{C}$ for $24 \mathrm{~h}$. The dye concentration in each polymer was maintained low, typically being $c a .5 \mu \mathrm{M}$. The fluorescence lifetime of EB in polymer was measured by time-correlated single photon counting (TCSPC). The laser source for sample excitation was a picosecond diode laser operating at a wavelength of $467 \mathrm{~nm}$ at $10 \mathrm{MHz}$ (Picoquant). A dichroic mirror and a $488 \mathrm{~nm}$ long pass filter (Semrock) were mounted to collect the emission and to eliminate the excitation wavelength. With a fast detector (Hamamatsu R3809) and a TCSPC board (Becker-Hickl SPC-830), the instrument response function (IRF) of the system is typically about $150 \mathrm{ps}$. To avoid local bleaching in the polymer, the sample was continuously scanned at a rate of $1 \mathrm{~Hz}$ in the area of $100 \mu \mathrm{m} \times 100 \mu \mathrm{m}$, during the measurements.

Figure 2 shows the fluorescence decay curves of EB in five polymers. All data do not exhibit single exponential, reflecting the structural heterogeneity of the media. The fluorescence lifetimes were extracted from the measured decay curves by a nonlinear least square fit with deconvolution of the instrument response function. The curves were fit by a double exponential form and the average lifetime, defined as $\alpha_{1} \tau_{1}+\alpha_{2} \tau_{2} /\left(\alpha_{1}+\alpha_{2}\right)$, was obtained and shown in Table 1 . The average lifetime was used as a characteristic time constant that represents the excited state dynamics of EB in polymers. The average lifetime of EB was shortest in LDPE (2.12 ns) and longest in PAA (14.84 ns) with the order of LDPE $<$ HDPE $<$ PC $<$ PS $<$ PAA. If the hydrogen bonding effect is applicable, the EB lifetime in PAA should be shorter than that in LDPE. That is, considering the molecular structure of polymer, the trend has nothing to do with

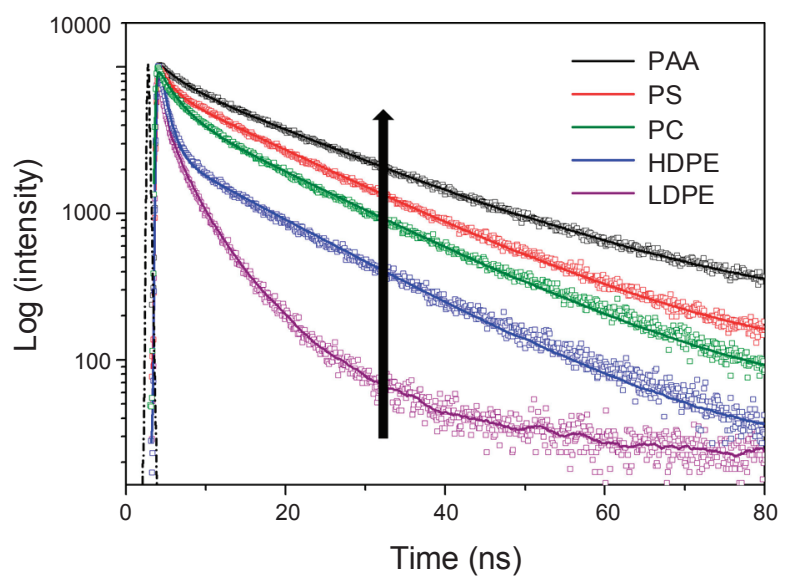

Figure 2. The fluorescence decay curves of ethidium bromide in five different polymers. 
Table 1. The fluorescence decay times of ethidium bromide in polymer. The average lifetime, $\langle\tau\rangle$, was obtained from the bi-exponential fit

\begin{tabular}{ccccrc}
\hline Polymer & $\alpha_{1}$ & $\tau_{1}(\mathrm{~ns})$ & $\alpha_{2}$ & $\tau_{2}(\mathrm{~ns})$ & $<\tau>(\mathrm{ns})$ \\
\hline LDPE & 0.77 & 0.86 & 0.23 & 6.34 & 2.12 \\
HDPE & 0.66 & 1.63 & 0.34 & 12.42 & 5.30 \\
PC & 0.47 & 2.70 & 0.53 & 15.66 & 9.57 \\
PS & 0.34 & 2.76 & 0.66 & 17.18 & 12.28 \\
PAA & 0.26 & 2.72 & 0.74 & 19.10 & 14.84 \\
\hline
\end{tabular}

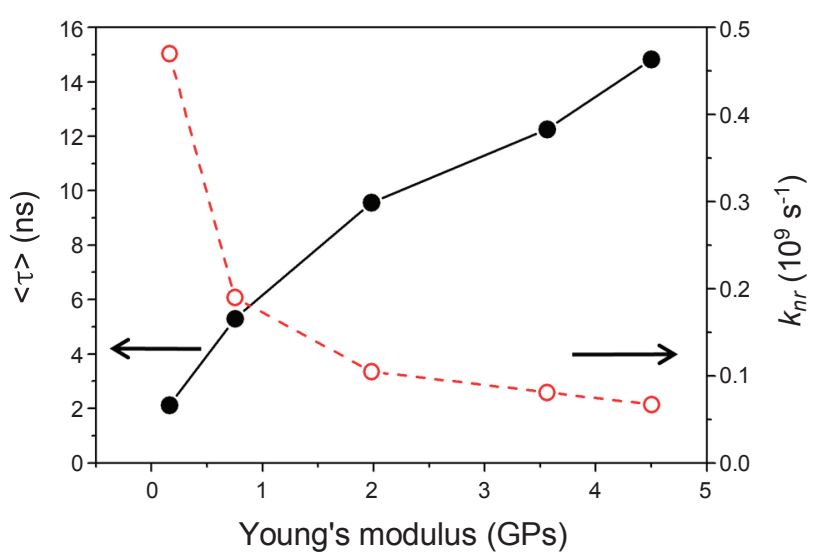

Figure 3. The fluorescence lifetime and the nonradiative rate constant of EB as a function of the Young's modulus of polymer.

the excited-state proton transfer. The fluorescence lifetime measurements of EB in rigid media are rare in the literature. The fluorescence lifetimes of EB in trehalose glasses containing $10-20 \%$ of water are in the range of $13.5-16.5 \mathrm{~ns} .^{6}$ The higher water content reduces the fluorescence lifetime. Bunker et al. measured the EB lifetime in Nafion polymer and observed a biexponential decay with 3.2 ns and 12 ns for the short and long components, respectively. ${ }^{7}$ Lee et al. measured the fluorescence decay dynamics of DNA-EB complex in polymer at the single molecular level. ${ }^{8}$ However, these reports did not concern the effect of polymer rigidity on the excited-state EB dynamics.

In Figure 2, we plotted the average lifetime of EB as a function of the Young's modulus of polymer. The Young's moduli of the five polymers were taken from the reported values in literature. ${ }^{9}$ The fluorescence enhancement of EB with increasing medium rigidity indicates that the internal molecular rotor dynamics of EB is affected by the mechanical property of poly- mer. The nonradiative decay rate constant can be obtained from the measured fluorescence lifetime by

$$
k_{n r}=\frac{1}{<\tau>}-k_{r}
$$

The radiative rate constant, $k_{r}$, of EB has been known as $1.4 \times$ $10^{7} \mathrm{~s}^{-1}$. The nonradiative rate constant was calculated by using Eq. (1) and plotted in Figure 3 as a function of the Young's modulus. Previously, we have reported that DND-189, a $\mathrm{pH}$ sensitive dye, can be used as a environmental rigidity sensor. ${ }^{10}$ This work showed that EB, a DNA intercalating dye, can also be used as a medium rigidity probe. Although it is very likely that the phenyl ring rotation is responsible for the excited-state dynamics of EB in polymer, further work may be required to clarify this issue more clearly.

In conclusion, the fluorescence lifetimes of EB in five polymers covering LDPE, HDPE, PC, PS, and PAA were measured by picosecond time-correlated single photon counting. The lifetime change of EB has been previously described by hydrogen bonding ability. In this work, we have observed that the lifetime of EB depends strongly on the Young's modulus of medium. Thus, it is possible that the fluorescence decay dynamics of EB could be influenced by medium rigidity rather than hydrogen bonding ability in polymer.

Acknowledgments. This work was supported by the Acceleration Research Program of the Korea Science and Engineering Foundation.

\section{References}

1. Olmsted, J., III.; Kearns, D. R. Biochemistry 1977, 16, 3647.

2. Collini, M.; Chirico, G.; Baldini, G. Biopolymers 1992, 32, 1447.

3. Pal, S. K.; Mandal, D.; Bhattacharyya, K. J. Phys. Chem. B 1998, 14, 185.

4. Dandliker, P. D.; Holmline, R. E.; Barton, J. K. Science 1997, 275 , 1465.

5. Serwer, P.; Hayes, S. J. Biophys. J. 2001, 81, 3398.

6. Collini, M.; D’Alfonso, L.; Baldini, G. Photochem. Photobiol. 2003, 77, 376.

7. Bunker, C. E.; Rollins, H. W.; Ma, B.; Simmons, K. J.; Liu, J.-T.; Ma, J.-J.; Martin, C. W.; DesMarteau, D. D.; Sun, Y.-P. J. Photochem. Photobiol. A: Chemistry 1999, 126, 71.

8. Lee, J.; Lee, J.; Lee, M.; Lee, K.-J.-B.; Ko, D.-S. Chem. Phys. Lett. 2004, 394, 49

9. Jee, A.-Y.; Lee, M. Polym. Test. 2010, 29, 95.

10. Kwon, H.; Jee, A.-Y.; Lee, M. Bull. Korean Chem. Soc. 2009, 30, 1237. 